\title{
Delimitación temática de la investigación española en fisioterapia a través del análisis de co-palabras
}

\author{
Thematic delimitation of the Spanish research in Physiotherapy through co-word analysis
}

Victoria Calvo fuente (1), Gisela Cantos Mateos (2), María Ángeles Zulueta García (3)

(1) Facultad de Enfermería y Fisioterapia, Universidad de Alcalá, Alcalá de Henares, España, victoria.calvo@uah.es (2) Instituto de Políticas y Bienes Públicos, Consejo Superior de Investigaciones Científicas, Madrid, España, Gisela.cantos@csic.es (3) Facultad de Documentación, Universidad de Alcalá, Alcalá de Henares, España, Zulueta@uah.es

\section{Resumen}

Se determina mediante análisis de co-palabras, los principales temas de investigación de la Fisioterapia en España mediante un estudio descriptivo longitudinal retrospectivo. Se recuperaron los documentos de la Web of Science que versan sobre rehabilitación y fisioterapia en España, sin límite de fechas de publicación; y se trataron con técnicas de análisis y visualización de redes para facilitar la interpretación gráfica de la información basada en coocurrencias de KeyWord Plus. Las principales líneas de investigación detectadas versan sobre rehabilitación y calidad de vida en enfermedades pulmonares, en enfermedades cardíacas, en reconstrucción articular, en lesiones neurológicas y sobre disfunciones del aparato locomotor y su abordaje a través de diferentes modalidades de fisioterapia. La metodología propuesta ha resultado interesante para hacer una primera aproximación a la delimitación temática de la fisioterapia. Futuros trabajos deberían enfocarse al estudio en profundidad de los descriptores más representativos sobre Disfunciones del aparato locomotor, para determinar los temas de investigación de la Fisioterapia en España de forma más específica.

Palabras clave: Rehabilitación. Fisioterapia. Frentes de investigación. España. Análisis de co-palabras.

\section{Introducción}

Desde que en el año 1957 se produjo la institucionalización de la fisioterapia en España hasta nuestros días, la actividad profesional de los fisioterapeutas españoles está envuelta en un sistema de cambio constante, pasando del importante protagonismo que ha supuesto hasta hace unos años la labor asistencial, al actual desarrollo de la actividad investigadora.

El crecimiento de la producción científica, así como su recopilación en bases de datos bibliográficas automatizadas, ha potenciado el uso de estudios bibliométricos que aportan una visión interesante de la actividad científica (Bordons y Zulueta, 1999).

\begin{abstract}
The main research topics of Physiotherapy in Spain were determined using co-word analysis in a retrospective longitudinal descriptive study. Documents that deal with Rehabilitation and Physiotherapy in Spain were retrieved from the Web of Science, without publication date limits; and analysis and network visualization techniques were used to facilitate the graphical interpretation of information, based on the co-occurrence analysis of KeyWord Plus. the main research lines are about rehabilitation and quality of life in lung diseases, heart diseases, in joint reconstruction, neurological injuries and musculoskeletal dysfunctions and its management through different physiotherapy modalities. The proposed method has turned out to be interesting for a first approach to the delimitation of Physiotherapy domain. Future works should focus on the depth study of the most representative descriptors in musculoskeletal dysfunctions to determine the research topics of Physiotherapy in Spain in a more specific way.
\end{abstract}

Keywords: Rehabilitation. Physiotherapy. Research fronts. Spain. Co-word analysis.

Para precisar la temática del análisis bibliométrico se puede optar por utilizar "palabras clave" como unidades de análisis, ya que permiten identificar con precisión el contenido intelectual de un documento científico (Vargas-Quesada et al., 2007). Las palabras clave que se extraen de los registros para este estudio son las KeyWord Plus (KWP) generadas por la base de datos.

El análisis de co-palabras está basado en la coocurrencia de términos extraídos habitualmente del título, resumen y palabras clave de una publicación científica. Describe redes en función de las frecuencias con las que se producen estas relaciones (Jacobs, 2002).

La visualización de estas relaciones se consigue a través de técnicas de representación gráfica 
de la información basadas en la teoría de las redes sociales.

El presente trabajo se centra en el análisis de coocurrencias de KWP de publicaciones recogidas en la Web of Science del Institute for Scientific Information (ISI)

El objetivo es determinar mediante análisis de co-palabras, los principales temas de investigación de la disciplina en estudio. Se complementa con un escueto análisis de revistas y categorías para concretar estos temas, dada la marcada multidisciplinariedad del dominio.

\section{Antecedentes}

La mayoría de los estudios bibliométricos sobre fisioterapia en España se centran en el análisis de la producción científica en las publicaciones de esta disciplina a nivel nacional (Massó et al., 2000 y 2001; Martínez et al., 2002 y 2003; Valera, 2007; Meroño, 2010).

La literatura muestra precedentes en el análisis de coocurrencias de palabras. Zulueta et al. (2011) muestran las líneas de investigación sobre salud y mujer a través de Medline, utilizando técnicas de visualización de redes y análisis factorial. Zhang et al. (2012) utilizan el análisis de co-palabras y de redes sociales para estudiar la investigación sobre adherencia de los pacientes a los tratamientos.

EI uso de las KWP como unidades de análisis se ha visto reflejado en los trabajos de Qin (2000) -que explora las similitudes y diferencias entre los descriptores del SCl y los términos MeSH- y de Cantos-Mateos et al. (2012) -que determinan las líneas de investigación española con células madre a través del análisis de KWP-.

\section{Material y métodos}

En este estudio se utiliza como fuente de datos la Web of Science del Institute for Scientific Information (ISI) Thomson Reuters.

Se realizó una búsqueda utilizando los términos Rehabilitation, Physical therapy y Physiotherapy en el campo topic, y Spain en el campo address, sin parametrización temporal.

Para la representación y visualización de datos bibliográficos recurrimos al programa VOSviewer 1.5.4, que permite identificar de forma rápida los principales campos de investigación de un dominio. Se seleccionó, por tanto, como método para obtener la delimitación temática el análisis de redes de información científica resultantes de la coocurrencia de las KWP en los documentos.

\section{Resultados}

Los documentos recuperados ascienden a 1.344 , de los que $1.175(87,5 \%)$ están indizados con KWP y 169 no. De los registros indizados se extraen $8.557 \mathrm{KWP}$, con una media por registro de 7,28 (Tabla I). De este cómputo global, resultan 3.883 KWP únicos, siendo el descriptor Rehabilitation el de frecuencia más elevada, seguido de Quality of life y Management (Tabla II).

\begin{tabular}{lc}
\hline Total de registros en la base de datos & 1344 \\
\hline Registros indizados & 1175 \\
\hline Registros no indizados & 169 \\
\hline Total de KWP & 8557 \\
\hline KWP únicos & 3883 \\
\hline KWP /Registro indizado & 7,28 \\
\hline
\end{tabular}

Tabla I. Datos generales

\begin{tabular}{lclc}
\hline Descriptores & Frecuencia & Descriptores & Frecuencia \\
\hline Rehabilitation & 233 & Exercise & 44 \\
\hline Quality-of-life & 90 & $\begin{array}{l}\text { Obstructive } \\
\text { pulmonary- } \\
\text { disease }\end{array}$ & 42 \\
\hline Management & 72 & Outcomes & 37 \\
\hline Recovery & 51 & Mortality & 37 \\
\hline Therapy & 51 & Performance & 37 \\
\hline $\begin{array}{l}\text { Randomized } \\
\text { controlled-trial }\end{array}$ & 50 & Double-blind & 31 \\
\hline Follow-up & 46 & Scale & 31 \\
\hline Disability & 45 & Health & 31 \\
\hline Reliability & 44 & Disease & 31 \\
\hline
\end{tabular}

Tabla II. Descriptores más frecuentes

\subsection{Revistas de publicación}

El análisis de revistas y su clasificación en categorías temáticas aporta información complementaria a la que proporcionan los KWP en la delimitación temática.

El número total de revistas en las que se publicaron los documentos es de 521 , lo que sugiere una notable multidisciplinariedad del dominio estudiado. Destaca la productividad de la Revista de Neurología (106 documentos) y Archives of Physical Medicine and Rehabilitation (78 documentos) que agrupan el $14 \%$ del total. 


\begin{tabular}{lcc}
\hline Revista & $N^{\circ}$ doc. & $\%$ doc. \\
\hline Revista de neurología & 106 & 7,9 \\
\hline $\begin{array}{l}\text { Archives of Physical Medicine and } \\
\text { Rehabilitation }\end{array}$ & 78 & 5,8 \\
\hline Archivos de bronconeumología & 31 & 2,3 \\
\hline Medicina clínica & 29 & 2,2 \\
\hline Revista española de cardiología & 22 & 1,6 \\
\hline Haemophilia & 21 & 1,6 \\
\hline Actas españolas de psiquiatría & 19 & 1,4 \\
\hline Neurología & 18 & 1,3 \\
\hline $\begin{array}{l}\text { Medicina oral patología oral y cirugía } \\
\text { bucal }\end{array}$ & 18 & 1,3 \\
\hline Brain injury & 17 & 1,3 \\
\hline
\end{tabular}

Tabla III. Revistas más productivas

\subsection{Análisis de categorías}

Los documentos se publicaron en revistas clasificadas en 134 categorías temáticas. Destacan especialmente Clinical Neurology y Rehabilitation que reúnen el $26 \%$ del total de documentos. Junto a ellas destacan también Sport Sciences y Neurosciences con 155 y 114 documentos respectivamente (Tabla IV).

\begin{tabular}{lcc}
\hline Categorias & $N^{\circ}$ doc. & $\%$ doc. \\
\hline Clinical Neurology & 265 & 13,3 \\
\hline Rehabilitation & 246 & 12,3 \\
\hline Sport Sciences & 155 & 7,8 \\
\hline Neurosciences & 114 & 5,7 \\
\hline Dentistry, Oral Surgery \& Medicine & 79 & 4,0 \\
\hline Respiratory System & 78 & 3,9 \\
\hline Orthopedics & 76 & 3,8 \\
\hline Surgery & 72 & 3,6 \\
\hline Medicine, General \& Internal & 69 & 3,5 \\
\hline
\end{tabular}

Tabla IV. Categorías temáticas más productivas

\subsection{Estudio de redes}

Con vistas a identificar los principales temas de investigación en rehabilitación y fisioterapia, se generaron dos visualizaciones distintas sobre un mismo mapa creado a partir de la matriz de coocurrencia de los KWP mediante el programa vOSviewer.

Como se aprecia en la Figura 1, los términos más destacados son centrales y están bastante próximos entre sí (rehabilitation y quality of life).

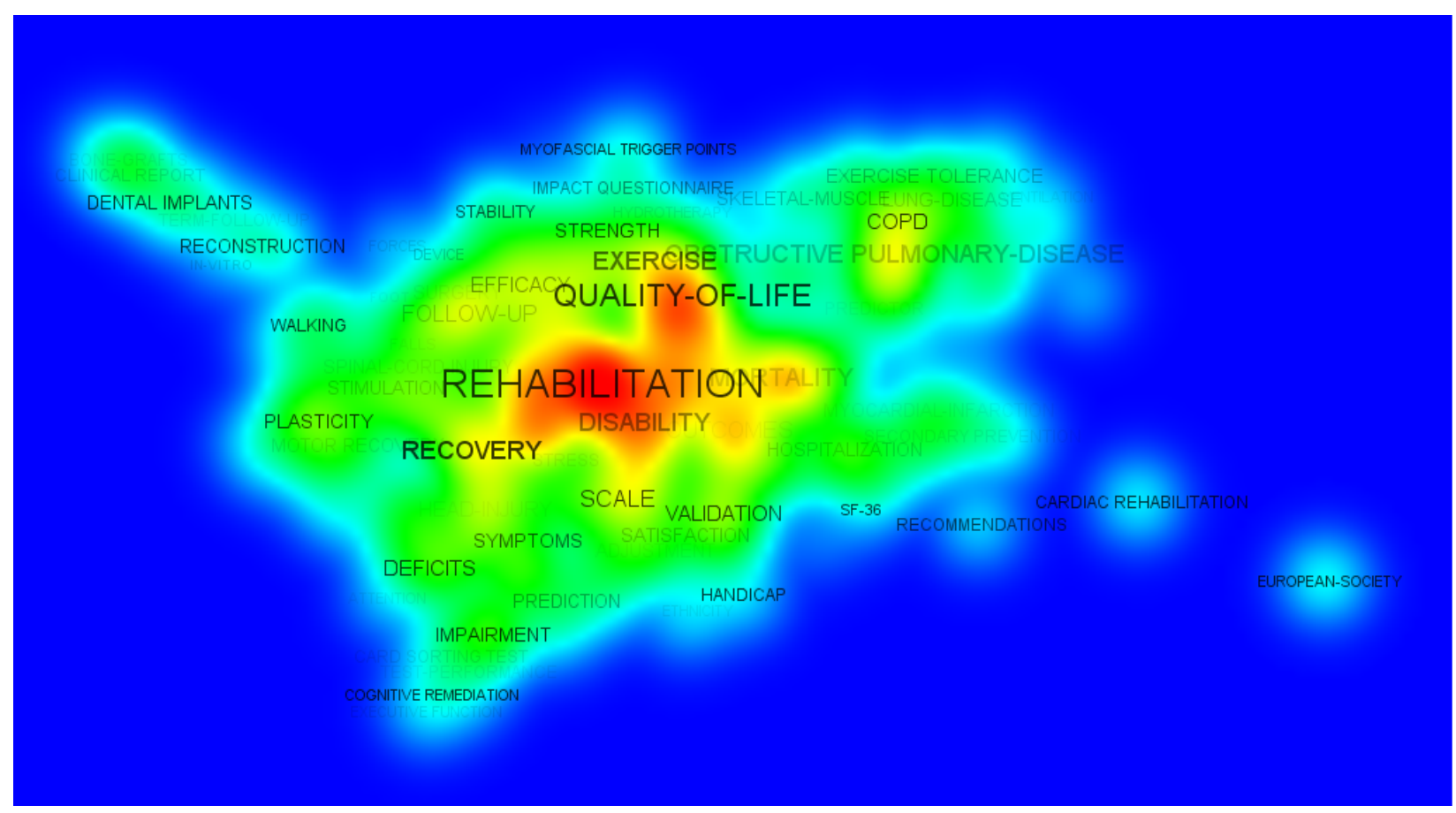

Figura 1. Visualización de líneas de investigación en rehabilitación y fisioterapia: mapa densidades 


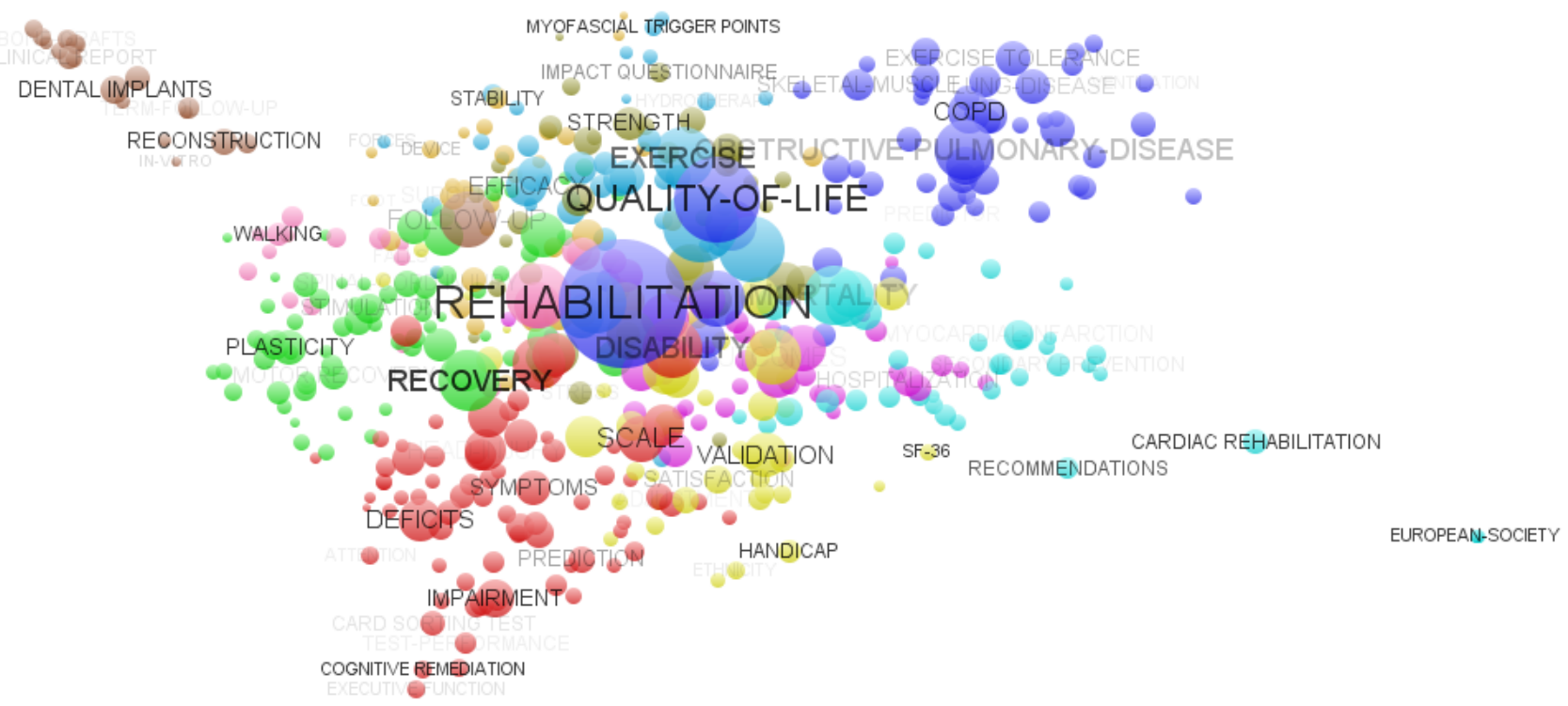

Figura 2. Visualización de líneas de investigación en rehabilitación y fisioterapia: mapa etiquetas

Hay otros términos menos destacados -exercise, disability y recovery- cercanos a los núcleos centrales, que sirven de nexo con otros subcampos. Las áreas de investigación no quedan claramente definidas, porque los KWP más frecuentes aparecen fuertemente agregados. Por ello, la estructura del mapa apunta a que el foco principal lo constituye la rehabilitación junto con calidad de vida.

Para precisar la existencia de estas líneas, el mismo mapa de términos (Figura 2) muestra ahora en clústers los diferentes temas de estudio. En la parte superior se representa la investigación sobre disfunciones del aparato locomotor y el manejo de las mismas con ejercicio y a través de diferentes modalidades de fisioterapia. A la izquierda se vislumbra la investigación sobre recuperación de lesiones neurológicas. En la parte inferior izquierda se representan los estudios sobre daño cerebral y enfermedades mentales. Abajo se aprecia una posible línea de estudios sobre epidemiología. A la derecha se representa la rehabilitación en enfermedades pulmonares y calidad de vida de estos sujetos, y en patología cardíaca. En la parte superior izquierda el seguimiento en reconstrucción articular y dental.

Se estima la existencia de tres líneas de investigación más consolidadas, correspondientes a la rehabilitación y calidad de vida en enfermedades pulmonares, en enfermedades cardíacas y la reconstrucción articular y dental. El resto de líneas podrían considerarse emergentes, como la que trata sobre disfunciones del aparato locomotor. Si bien la mayoría de temas reflejan la investigación en rehabilitación, es evidente que en todos hay representada una parte de la fisioterapia, y es este último el que muestra un vínculo más estrecho con esta disciplina.

\section{Conclusiones}

Los resultados obtenidos perfilan la imagen de la investigación española en rehabilitación. Dentro de la misma podría estar representada la fisioterapia, dado el marcado carácter multidisciplinar de la rehabilitación y la estrecha relación entre ambas disciplinas. Esta multidisciplinariedad queda asimismo reflejada en la diversidad de categorías resultantes en las que se clasifican las publicaciones estudiadas.

La metodología propuesta ha resultado interesante para hacer una primera aproximación a la delimitación temática de la fisioterapia. Futuros trabajos deberían enfocarse al estudio en profundidad de los descriptores más representativos en la línea de disfunciones del aparato locomotor, para determinar los temas de investigación de la fisioterapia en España de forma más específica.

\section{Referencias}

Bordons, M.; Zulueta, M. A. (1999). Evaluación de la actividad científica a través de indicadores bibliométricos. // Revista Española de Cardiología. 52:10 (1999) 790-800.

Cantos-Mateos, G.; Vargas-Quesada, B.; ChinchillaRodríguez, Z.; Zulueta, M. A. (2012). Stem cell research: Bibliometric analysis of main research areas through KeyWords Plus. // Aslib Proceedings. 64:6 (2012) 561590 . 
Jacobs N. (2002). Co-term network analysis as a means of describing the information landscapes of knowledge communities across sectors. // Journal of Documentation. $58: 5$ (2002) 548-562.

Martínez, M.; Marín, J.; Rebollo, J. (2002). Producción científica de la revista Cuestiones de Fisioterapia (19952000). // Cuestiones de Fisioterapia. 20:1 (2002) 1-11.

Martínez, M.; Gómez, A. (2003). Estudio bibliométrico de la Revista Iberoamericana de Fisioterapia y Kinesiología (1998-2002). // Revista Iberoamericana de Fisioterapia y Kinesiología. 6:1 (2003) 58-71.

Massó, J. J.; Bernabéu, M.; Medina, F. (2000). Productividad de los fisioterapeutas españoles en el período 19911999 a través del análisis bibliométrico de la revista Fisioterapia. // Fisioterapia. 22:1 (2000) 2-11.

Massó, J. J.; Valera, J. F.; Medina, F.; Bernabeu, M. (2001). Análisis del consumo de información en la revista Fisioterapia (1991-1999). // Fisioterapia. 23:3 (2001) 154159

Meroño, A. J. (2010). Desarrollo científico de la Fisioterapia en España. Estudio de los artículos publicados en la Revista Fisioterapia (1979-2006). Sevilla: Universidad, 2010. Tesis Doctoral.

Qin, J. (2000). Semantic similarities between a keyword database and a controlled vocabulary database: An investigation in the antibiotic resistance literature. //
Journal of the American Society for Information Science. $51: 2$ (2000) 166-180.

Valera, J. F. (2007). La investigación en Fisioterapia en España a través del análisis bibliométrico de la Revista Fisioterapia (1991-1999). Murcia: Universidad, 2010. Tesis Doctoral

Vargas-Quesada, B.; Doménech, I.; García, G.; Sánchez, C.; Extremeño, A.; Zulueta, M. A. (2007). La identificación temática a partir de la visualización de la información: una aproximación mediante el caso de women en Medline. // Revista Española de Documentación Científica. 30:2 (2007) 163-177.

Zhang, J.; Xie, J.; Hou, W. L.; Tu, X. C.; Xu, J.; Song, F. J.; et al. (2012). Mapping the Knowledge Structure of Research on Patient Adherence: Knowledge Domain Visualization Based Co-Word Analysis and Social Network Analysis. // Plos one. 7:4 (2012) e34497.

Zulueta, MA; Cantos-Mateos, G; Vargas-Quesada, B; Sánchez, C. (2011). Research involving women and health in the Medline database, 1965-2005: co-term analysis and visualization of main lines of research. // Scientometrics. 88:3(2011):679-706.

Enviado: 2013-04-05. Segunda versión: 2013-08-22. Aceptado: 2013-09-05. 
